\title{
Identification of differentially expressed microRNAs under imidacloprid exposure in Sitobion avenae Fabricius
}

\section{Baizhong Zhang}

Henan Institute of Science and Technology

\section{Xu Su}

Henan Institute of Science and Technology

\section{Lanfen Xie}

Henan Institute of Science and Technology

Wen-Yang Dong

China Agricultural University

\section{Fan-Bin Kong}

Henan Institute of Science and Technology

Junjie Liu

Henan Instiute of Science and Technology

Liuyang Lu

Henan Institute of Science and Technology

\section{Ya-She Li}

Henan Institute of Science and Technology

\section{Shouping Zhang}

Henan Institute of Science and Technology

\section{Ming-Wang Shi}

Henan Institute of Science and Technology

Xi-Ling Chen ( $\square$ chenxiling456@126.com )

Henan Institute of Science and Technology

\section{Xi-Wu Gao}

China Agricultural University

\section{Research article}

Keywords: MicroRNAs (miRNAs), Sitobion avenae, imidacloprid, resistance

Posted Date: October 24th, 2019

DOI: https://doi.org/10.21203/rs.2.9874/v2 
License: (c) (i) This work is licensed under a Creative Commons Attribution 4.0 International License. Read Full License 


\section{Abstract}

Background MicroRNAs (miRNAs), which are short single-stranded non-coding RNAs, regulate the expression of target genes, especially those involved in the regulation or metabolism of endogenous or xenobiotic compounds. Results De novo assemblies of the transcriptome of Sitobion avenae Fabricius under control conditions and under imidacloprid treatment were obtained using Illumina short-read sequencing technology. Fifty-seven miRNAs, of which 36 were known and 21 were novel, were identified. Quantitative analysis of miRNA levels showed that five miRNAs were significantly up-regulated, and 11 miRNAs were significantly down-regulated in the nymphs of $\mathrm{S}$. avenae treated with imidacloprid in comparison with those of the control. Analysis of the candidate target genes in S. avenae that could be regulated by these miRNAs were also carried out. The functions of the miRNAs, which could potentially regulate target genes that participate in metabolism, regulatory or detoxification pathways in S. avenae, were clarified based on Gene Ontology and KEGG pathway analysis. The effects of the miRNAs api-miR1000, api-miR-316, and api-miR-iab-4 on susceptibility of S. avenae to imidacloprid was determined. Modulation of the abundances of api-miR-1000, api-miR-316, and api-miR-iab-4 by the addition of the correspondign inhibitors to the artificial diet significantly changed the susceptibility of S. avenae to imidacloprid, which further demonstrated the effect of these miRNAs on the regulation or metabolism of insecticides.Conclusion The results of this study suggested that miRNAs differentially expressed in response to imidacloprid could play a critical regulatory role in the resistance of $\mathrm{S}$. avenae to imidacloprid.

\section{Background}

MicroRNAs (miRNAs) are between 18 and 23 nucleotides in length and have been shown to be responsible for the post-transcriptional regulation of mRNAs. miRNAs can silence expression of target genes by complementarily binding to $5^{\prime} / 3^{\prime}$ untranslated regions (UTRs) or coding sequences. ${ }^{1-5}$ UTRs of target mRNAs are mainly bound through imperfect complementary base pairing. ${ }^{6}$ The sequence of the target is an exact match to the miRNA. ${ }^{7}$

miRNAs have crucial roles in development in many organisms and regulate most mammalian proteincoding genes, ${ }^{8}$ which makes them critical mediators in a series of physiological and developmental pathways, ${ }^{9-12}$ Such as apoptosis, cell proliferation, morphogenesis, and the metabolism of compounds. ${ }^{5 \text {, }}$ 13

MiRNAs can mediate the detoxification/metabolism of xenobiotics through regulation of the expression of detoxification genes, including nuclear receptors and xenobiotic-metabolizing enzymes. ${ }^{5}$ For example, human UDP-glucuronosyltransferase (UGT) 1A, which metabolizes raloxifene metabolites, was shown to be regulated post-transcriptionally by mir-491-3p, ${ }^{14}$ and human P450 CYP1A1, which is associated with the metabolism of carcinogenic and metabolites, can be regulated post-transcriptionally by miR-892a. ${ }^{15}$ 
Previous studies conducted in Plutella xylostella indicated that miRNAs could be involved in chlorantraniliprole resistance by regulating the expression levels of UGT genes. ${ }^{16}$ UGT2B7 and UGT2B15 were shown to be regulated post-transcriptionally by miRNAs; ${ }^{17}$ Culex pipiens miRNAs are involved in pyrethroid resistance by regulating the expression levels of P450 genes. ${ }^{18,19}$ There are many miRNAs known to be involved in regulating enzymes that metabolize xenobiotics in animals, while the effects of miRNAs on the regulation of xenobiotic detoxification/metabolism in wheat aphids is less understood.

The grain aphid Sitobion avenae is the main pest of wheat and is distributed worldwide. S. avenaenot only harms crops by sucking juice from plants but also spreads yellow dwarf virus disease, leading to serious economic losses. The control of this aphid has depended largely on the use of chemically synthesized pesticides, ${ }^{20}$ such as imidacloprid, which is a neonicotinoid that targets sucking pests, such as aphids and the green leaf bug. ${ }^{21}$ It has been widely applied in wheat fields to control wheat aphid in China. As a result, $S$. avenae has developed serious resistance to imidacloprid. ${ }^{22}$

Here, we performed high-throughput sequencing of short RNA libraries of control and imidacloprid-treated $S$. avenae nymphs to detect differentially expressed microRNAs, and determine the functions of miRNAs in regulating genes involved in the metabolism/detoxification of xenobiotics. The results of this study will help increase our understanding of the roles of miRNAs in the regulation of resistance to imidacloprid in insects.

\section{Methods}

\subsection{Culture conditions}

The susceptible strain of $S$. avenae used was maintained in a greenhouse for more than 10 years and has never been exposed to any insecticides. The culture conditions used were described previously. ${ }^{23}$

\subsection{Imidacloprid treatments}

To test the susceptibility of $S$. avenae to imidacloprid, the leaf-dipping method was conducted as previously described by Chen et al. ${ }^{24}$ with minor modifications. Imidacloprid was dissolved in acetone, and then serially diluted with $0.05 \%(\mathrm{v} / \mathrm{v})$ Triton X-100 in water. Wheat leaves were cut into pieces $20 \mathrm{~mm}$ long. The leaves with aphids were dipped into $0.05 \%$ (v/v) Triton X-100 in water with $0.5 \mathrm{mg} / \mathrm{L}$ imidacloprid ( $\mathrm{LC}_{10}$ concentration, Table S1) or without imidacloprid as a control. The leaves with treated aphids were put into a glass tube ( $6 \mathrm{~cm}$ in length, $2 \mathrm{~cm}$ diameter) and the open end was covered with cotton to prevent insect escape. Surviving third instar nymphs ( $\mathrm{N}=5$ for each treatment) were collected at $24 \mathrm{~h}$ after imidacloprid or mock challenge. Aphids were flash frozen in liquid nitrogen before being stored at $-80^{\circ} \mathrm{C}$ for RNA extraction. The experiment was replicated three times.

\subsection{RNA isolation, miRNA library construction and Illumina sequencing}


Total RNA was extracted from control and imidacloprid-treated $S$. avenae using TRIzol reagent (Invitrogen, Shanghai, China) according to the manufacturer's protocol and resuspended in nuclease-free water. Electrophoresis on $1 \%$ agarose gels was used to determine if the extracted RNA was degraded or contaminated, and then purity, concentration and integrity of the RNA were measured using a NanoDrop 2000 spectrophotometer (Thermo Scientific, Wilmington, DE), a Qubit® RNA Assay Kit with a Qubit ${ }^{\circledR} 2.0$ Fluorometer (Life Technologies, CA, USA), and a RNA Nano 6000 Assay Kit with the Agilent Bioanalyzer 2100 system (Agilent Technologies, CA, USA), respectively. Approximately $3 \mu \mathrm{g}$ of total RNA from each sample was used as input material for constructing the small RNA library, and the platform used for sequencing was conducted in Novogene, Beijing, China as previously described. ${ }^{25}$

\subsection{Bioinformatics analysis, miRNA prediction, and miRNA target prediction.}

S. avenae miRNAs, were confirmed using miRDeep2 software. ${ }^{26}$ Raw reads from the four libraries (imidacloprid treatment and control, two biological replicates each) were used as input for miRDeep2, and the data from each library were separately analyzed. The default options and settings were used to perform the miRDeep2 analysis. The raw read sequences with polyA tails and miRNAs with lengths ranging from 18 to $30 \mathrm{nt}$ were selected for further analysis after trimming adaptor sequences and removing snRNA, rRNA, snoRNA and tRNA sequences. The sequences that mapped to the predicted mature Acythosiphon pisum miRNAs annotated in miRBase were identified as known mature miRNAs. The clean reads from the $S$. avenae transcriptome have been deposited in the NCBI/SRA database under accession number SRP182781. The Miranda, RNAhybrid, and Target Scan programs were used to predict the target gene of the miRNAs according to the miRNA-binding sites. ${ }^{27-29}$ Then only the binding sites commonly identified by the three tools were selected for subsequent analysis. The putative miRNA targets were used as queries in searches against the $S$. avenae transcriptome sequences.

The predicted miRNA target genes were selected for further analysis. The predicted target genes were aligned using the NCBI BLASTX program, and mapping and annotation of the gene sequences were conducted using BLAST2GO. ${ }^{30}$ The Gene Ontology (GO) and Kyoto Encyclopedia of Genes and Genomes (KEGG) databases were used to further analyze the putative target genes of the miRNAs.

\section{5 miRNAs differentially expressed between control and imidacloprid-treated S. avenae}

In order to identify the aphid miRNAs that were affected by imidacloprid, the differences in the expression levels of miRNAs, as assessed by miRDeep2, between control and imidacloprid-treated $S$. avenae were determined. The edgeR software package (3.10.2) obtained from Bioconductor version 3.1 was used to analyze the read counts of the identified miRNAs

(http://www.bioconductor.org/packages/release/bioc/html/edgeR.html). ${ }^{31}$ edgeR is a Bioconductor software package for analyzing the differential expression of digital

gene expression data. The P-values were calculated using the Benjamini-Hochberg method. ${ }^{32} \mathrm{~A}$ corrected P-value of 0.05 was set as the default threshold for significance. Normalization of miRNA counts 
between libraries of the controls and treatments was executed according to the total number of reads across libraries. Normalized expression $=$ Actual miRNA count/Total count of clean reads $\times 10^{6}$. A fold change in miRNA expression $\geq 2$ with a false discovery rate $(F D R)<0.05$ was deemed to be significant.

\subsection{Quantitative real-time PCR (qPCR) validation}

To validate the miRNA data obtained by deep sequencing, the expression levels of three miRNAs were confirmed by qPCR. First strand cDNA was synthesized from $2 \mu \mathrm{g}$ of total RNA using the miScript II RT kit (Qiagen) following the manufacturer's instructions. The SYBR Green Master Mix (miScript SYBR Green PCR Kit, Qiagen) was used for miRNA expression assays, and qPCR was performed

as previously described. ${ }^{33}$ Relative expression was calculated using the $2^{-\Delta \Delta C t}$ method. ${ }^{34}$ U6 snRNA was used to normalize the expression levels of miRNAs as an endogenous control. The primers used are shown in Table 1.

\subsection{MiRNA inhibitor feeding in vitro, and the subsequent impacts on imidacloprid susceptibility}

The rearing method and the artificial diet used were the same as previously described. ${ }^{35} \mathrm{~A}$ sterilized transparent glass tube with both ends open was used as the feeding device $(4 \mathrm{~cm}$ in length, $2.5 \mathrm{~cm}$ diameter). The artificial diet, $25 \%$ sucrose, was put between the two layers of parafilm were sealed or was the parafilm containing the artificial diet used to seal one end of the feeding device, and then healthy apterous adults were transferred to the device; the ends were covered with mesh to prevent the insects from escaping. Each sample contained three replications.

To evaluate the inhibitory effect of the miRNAs on CYP6A14, 50 healthy apterous aphids were fed an artificial diet containing miRNA inhibitors at a final concentration of $2.5 \mathrm{mM} / \mathrm{L}$. The NC-inhibitor (a negative control) was used as the control. Following feeding for $24 \mathrm{~h}$, the surviving aphids were collected for RNA extraction. There were three replicates for each sample.

To evaluate the effects of miRNAs on the sensitivity of $S$. avenae to imidacloprid, 50 healthy apterous adults were fed an artificial diet that contained imidacloprid (at the $\mathrm{LC}_{50}$ concentration, $1.5 \mathrm{mg} / \mathrm{L}$ ) with an miRNA inhibitor $(2.5 \mathrm{mM} / \mathrm{L})$. The NC-inhibitor was used as the control. Three replicates were carried out, and mortality was scored at $48 \mathrm{~h}$. The miRNA inhibitors used were provided by Sangon Biotech Co., Ltd (Shanghai, China).

\section{Results}

\subsection{Illumina sequencing data analysis}

A total of $14,841,584$ raw reads were obtained from sequencing the control library and 13,964,533 raw reads were obtained from the imidacloprid treatment library (Table 2). The lengths of the S. avenae sRNAs ranged between $18 \mathrm{nt}$ and $35 \mathrm{nt}$, comprising $5.28 \%$ of the imidacloprid reads and $5.08 \%$ of the control reads were $22 \mathrm{nt}$ in size (Fig. 1A). After discarding low quality reads and adapter sequences, 
$6,992,451$ ( $47.11 \%$ of total) and 6,349,363 (45.47\%) unique mappable reads remained from the control and imidacloprid treatment libraries, respectively (Table 2). The length distribution of these mappable reads indicated that $3.75 \%$ of the total reads for the control and imidacloprid treatment, respectively, were $22 \mathrm{nt}$ in length and $5.18 \%$ and $6.23 \%$ of the total reads, respectively, were $23 \mathrm{nt}$ in length. The maximum lengths of reads from the imidacloprid and control libraries were $32 \mathrm{nt}(8.59 \%$ of all reads) and $34 \mathrm{nt}$ (9.76\%), respectively, these reads are likely piRNA-like sRNAs in S. avenae. A few sRNAs had over a thousand reads; however, most had fewer than 10 copies (Fig. 1C).

The dominance of uracil $(U)$ at the first position of the $5^{\prime}$ terminus is considered to be a conserved feature of mature miRNAs. Given that the first base of the 5 ' end of miRNAs is known to play very important roles in the interaction between miRNAs and Argonaute complexes, the position-specific occurrence of nucleotides in the candidate miRNA sequences was analyzed. S. avenae miRNAs showed a bias towards $U$ at the first nucleotide position; sRNAs showed a significant bias for $U$ at the $5^{\prime}(96.99 \%$, and $97.51 \%$, respectively) and $3^{\prime}$ ends (53.38\%, and $55.32 \%$, respectively) in both libraries. The nucleotide $U$ was the most abundant nucleotide at most of the positions. This higher abundance of $U$ was significant at positions $1,6,9,12,16$, and 22 based on analysis of the base composition of the miRNAs at each position.

\subsection{Sequence and prediction of miRNAs}

According to the distribution of read lengths, $22-$ nt miRNAs accounted for $43.86 \%$ of all species of miRNAs (Fig. 1D). The putative miRNAs in S. avenae were used as queries in searches against the miRNAs from the A. pisum genome in miRBase, and 36 conserved sequences were identified (Table S2). In addition, using the filtered S. avenae reads as queries in searches against mature miRNAs identified 26 miRNA sequence families from the miRBase database (Fig. 3A). It is predicted that the miR-10 family has the largest number of miRNAs $(n=415)$, followed by let-7 $(n=401)$, and miR-2 $(n=180)$. Insects were shared with these miRNAs, with the majority of miRNAs shared with the known A. pisum miRNAs, while a fewer extent miRNAs trended for other insect orthologs (Fig. 3B).

A total of 21 potentially novel miRNAs were identified; these were named with the prefix 'PC' (predicted candidate) based on the adopted nomenclature (Table S3). These novel miRNAs could be mapped to $S$. avenae transcriptome sequences. Mfold predictions revealed stem-loop hairpin secondary structures ( $<18$ $\left.\mathrm{kcal} / \mathrm{mole}^{36}\right)$ that were consistent with miRNA precursor sequences. Some of the predicted structures are shown in Fig. S1.

Differential expression of miRNAs between the imidacloprid treatments and controls was also determined according to normalized differences between Illumina read counts. Of the 57 S. avenae miRNAs, 16 $(28.1 \%)$ were differentially expressed, 11 of which were down-regulated and 5 of which were up-regulated (Table 3).

The PCR amplification products of api-miR-1000, api-miR-316, and api-miR-iab-4 were single bands with the expected sizes between 60 and $100 \mathrm{bp}$. Subsequent qPCR analysis confirmed that the expression 
level of api-miR-1000 was 5.5- fold higher in the imidacloprid treatment samples than in the controls. The expression levels of api-miR-316, and api-miR-iab-4 were $91 \%$, and $88 \%$ lower, respectively, in the imidacloprid treatment samples compared with the controls (Fig. S2). Overall, the results of qPCR analysis were similar to those of RNA-seq.

\subsection{Prediction of miRNA targets.}

The hypothetical miRNA target sequences within the UTRs of transcripts from S. avenae (including the targets of the 16 differentially expressed miRNAs) were predicted using Target Scan software (Table S4). To identify the possible functions of these hypothetical target genes, $\mathrm{GO}$ enrichment analysis was performed; target genes were assigned molecular function, biological process and cellular component terms (Table S5). The predicted miRNA target genes were annotated to $58 \mathrm{GO}$ level 2 terms(Fig. 4A), like some of these terms are related to metabolic processes.

In KEGG enrichment analysis miRNA target genes were assigned to 71 metabolic pathways, indicating that these pathways might be targeted by miRNA regulation (Fig. 4B). The orthologs of some miRNA targets are involved in biological pathways such as xenobiotic metabolism, indicating that miRNAs might be involved in the posttranscriptional regulation of these pathways (Table S6).

To confirm the potential function of miRNAs in the resistance of $S$. avenae to imidacloprid, the predicted target genes of the miRNAs that might participate in xenobiotic metabolism were further focused on. Interestingly, target genes for some miRNAs, such as those encoding cytochrome P450s, UDPglucuronosyltransferase and glutathione S-transferase, have been found to play critical roles in insect responses to xenobiotics (Table 4). Some miRNAs have many putative target genes, and many putative target genes are potentially regulated by multiple miRNAs (Table S3).

\subsection{Modulation of miRNAs impacts the expression of predicted target genes and the susceptibility of $S$. avenae to imidacloprid}

The expression levels of miRNAs miR-1000, miR-316, and miR-ab-4 were significantly lower in $S$. avenae adults fed an artificial diet containing the corresponding miRNA inhibitors than in adults fed the DEPC water control or NC control, The depression efficiencies of miR-1000, miR-316, and miR-ab-4 reached $85.0 \%, 89.1 \%$, and $88.2 \%$, respectively (Fig. $5 \mathrm{~A}$ ). The transcriptional levels of CYP6A14 were dramatically increased by 2.48 -fold in the miR-306-knockdown aphids. However, inhibition of the other two miRNAs had no significant effect on CYP6A14 transcript levels (Fig. 5B). Under imidacloprid stress, mortality decreased by $21.73 \%$ in the aphids fed miR-1000 inhibitors compared with those fed the controls. In contrast, mortality increased by $26.75 \%$ and $21.86 \%$ in the aphids fed miR-316 and miR-ab-4 inhibitors, respectively, compared with those fed the controls (Fig. 5C).

\section{Discussion}


It is predicted miRNAs can regulate majority of of protein-coding genes. ${ }^{37-39}$ It has also been demonstrated that miRNAs are involved in the regulation of various biological processes. In this study, miRNAs in $S$. avenae that might play regulatory roles in target gene responses to imidacloprid were identified and assessed. The identification and functional analysis of miRNAs differentially expressed in imidacloprid-treated $S$. avenae were conducted to increase our understanding of the regulation/detoxification of imidacloprid in insects. So far, high-throughput sequencing of small RNAs has been widely used to obtain miRNAs from a series of organisms, including S. avenae. Li et al. ${ }^{40}$ identified 345 miRNAs from winged and wingless $S$. avenae, whereas we only identified 57 miRNAs in control and imidacloprid-treated $S$. avenae as we only selected Acyrthosiphon pisum as a reference. However, our sequencing data greatly enlarges the range of available information about the involvement of miRNAs in the regulation of metabolism genes and provide a critical basis for further studying miRNAs related to insecticide resistance in $S$. avenae.

Our analysis of read counts indicated that 16 miRNAs were differentially expressed between imidaclopridtreated and control $S$. avenae, indicating that imidacloprid could modulate the expression of miRNAs and suggesting a probable regulatory role of these miRNAs in the detoxication/metabolism of insecticides in S. avenae. Several miRNAs up/down-regulated by imidacloprid treatment, e.g., api-miR-1000, api-miR-316, and api-miR-iab-4, could be involved in the regulation of imidacloprid metabolism. The activity-dependent expression of Drosophila melanogaster miR-1000 could indicate a mechanism for allowing neuronal activity to fine-tune the strength of excitatory synaptic transmission, and regulate the expression level of vesicular glutamate transporters, and load the glutamate into synaptic vesicles. ${ }^{41} \mathrm{~A}$ target site of D. melanogaster dme-miR-1000 is located in the intron of musashi, ${ }^{42}$ which encodes an RNA-binding protein, that regulates gene translation by prior expression in the nervous system. ${ }^{43}$ Honeybee miR-1000 is also thought to be involved in musashi function in the nervous system. ${ }^{44}$ The first miR-1000 identified in insects, and is expressed in a highly selective manner in insect brains. One cytochrome P450 and two glutathione S-transferase genes could be recognized by mse-miR-316. ${ }^{45}$ The accumulation of endogenous Ubx protein could be attenuated by ectopic expression of miR-iab-4-5p, and a classical homeotic mutant phenotype could also be induced (the transformation of halteres into wings). ${ }^{46}$ Drosophila dme-miR-iab-4 and dme-miR-iab-8 are miRNAs targeting bithorax-complex (BX-C) HOX bunches and participate in the regulation of reproduction and neural patterning through suppression of HOX gene targets. ${ }^{47}$

In order to further explore the role of miRNAs in the regulation of imidacloprid metabolism, the hypothetical target genes for the miRNAs identified were predicted, and many of them were annotated to biological processes. The target genes of several miRNAs from different families were previously shown to be involved in xenobiotic detoxication/metabolism. ${ }^{48-51}$ By combining differential analysis with target gene prediction, we also found that several of the miRNAs targeting genes putatively involved in xenobiotic detoxication/metabolism were expressed differentially between the imidacloprid-treated and control S. avenae. S. avenae CYP6A14 could be regulated by api-miR-316 in our present study. Furthermore, the mortality of $S$. avenae exposed to imidacloprid significantly decreased after being fed an 
api-miR-1000 inhibitor. In contrast, the mortality of $S$. avenae exposed to imidacloprid significantly increased after being fed an api-miR-316 or api-miR-iab-4 inhibitor. The differential expression of let-7 and miR-100 might be attributable to the influence of insecticides. A similar phenomenon was also observed in the host adaption of Myzus persicae to nicotianae, where let-7 and miR-100 participated in the posttranscriptional regulation of the expression of $\mathrm{CYP} 6 \mathrm{CY}$. Our results show shows that differentially expressed miRNAs may participate in the metabolism of imidacloprid by modulating the expression of genes related to xenobiotic metabolism.

In conclusion, of 57 miRNAs identified in S. avenae, the transcript levels of 16 were differentially regulated by imidacloprid treatment. The prediction of target genes related to xenobiotic metabolism showed that differentially expressed miRNAs could participate in the detoxication/metabolism of imidacloprid. The data presented here represent a crucial new genomics resource for further study of small RNAs in $S$. avenae.

\section{Abbreviations}

miRNAs: MicroRNAs; qPCR: Quantitative real-time PCR; BLASTX: Similarity search of the NCBI protein database using a translated nucleotide query; GO: Gene ontology; KEGG: Kyoto Encyclopedia of Genes and Genomes; nr: NCBI non-redundant protein sequences database; nt: NCBI nucleotide collection; UTRs: untranslated regions; SRA: Sequence Read Archive.

\section{Declarations}

\section{Acknowledgements}

This work is supported by the National Key Research and Development Program of China (2017YFD0201700), the Key Science and Technology Program of Henan (Agriculture) (182102110053), and Project of Plant Protection Key Discipline of Henan Province (1070202190011005).

\section{Funding}

Not applicable

\section{Availability of data and materials}

The transcriptome clean reads and computationally assembled sequences from this study were submitted to the NCBI/SRA database under accession number: SRX374716. The file for edgeR is the file 1.

\section{Author contributions statement}

X.G and X.C conceived and designed the experiments, B.Z., and J.L. performed the experiments, B.Z., X.S., S.Z., and X.G. analyzed the data and wrote the manuscript. J.L., and L.L. participated in the data analysis. 
All authors reviewed the manuscript.

\section{Competing interest}

The authors declare that they have no competing interests.

\section{Consent for publication}

Not applicable.

\section{Ethics approval and consent to participate}

Not applicable.

\section{Author details}

College of Resources and Environment, College of Animal Science and Veterinary Medicine, Henan Institute of Science and Technology, Xinxiang 453003, P.R. China

\section{References}

1. Asgari S, MicroRNA functions in insects. Insect Biochemistry and Molecular Biology 43: 388-397 (2013).

2. Pritchard CC, Cheng HH and Tewari M, MicroRNA profiling: approaches and considerations. Nature Reviews Genetics 13: 358-369.

3. Yokoi T and Nakajima M, MicroRNAs as mediators of drug toxicity. Annual Review of Pharmacology and Toxicology 53: 377-400 (2013).

4. Bartel DP, MicroRNAs: target recognition and regulatory functions. Cell 136: 215-33 (2009).

5. Rigoutsos I, New tricks for animal microRNAS: targeting of amino acid coding regions at conserved and nonconserved sites. Cancer Research 69: 3245-8 (2009).

6. Stark A, Kheradpour P, Parts L, Brennecke J, Hodges E, Hannon GJ and Kellis M, Systematic discovery and characterization of fly microRNAs using 12 Drosophila genomes. Genome research17: 18651879 (2007).

7. Wang X. Composition of seed sequence is a major determinant of microRNA targeting patterns. Bioinformatics 15, 1377-1383 (2014).

8. Krol J, Loedige I and Filipowicz W, The widespread regulation of microRNA biogenesis, function and decay. Nature Reviews Genetics 11: 597-610 (2010).

9. Suh N, Baehner L, Moltzahn F, Melton C, Shenoy A, Chen J and Blelloch R, MicroRNA function is globally suppressed in mouse oocytes and early embryos. Current Biology20: 271-277 (2010).

10. Xu C, Lu Y, Pan Z, Chu W, Luo X, Lin H, Xiao J, Shan H, Wang Z and Yang B, The muscle-specific microRNAs miR-1 and miR-133 produce opposing effects on apoptosis by targeting HSP60, HSP70 
and caspase-9 in cardiomyocytes. Journal of Cell Science120: 3045-3052 (2007).

11. Burnside J, Ouyang M, Anderson A, Bernberg E, Lu C, Meyers BC, Green PJ, Markis M, Isaacs G, Huang E and Morgan RW, Deep sequencing of chicken microRNAs. BMC Genomics 9: 185 (2008).

12. Legeai F, Rizk G, Walsh T, Edwards O, Gordon K, Lavenier D, Leterme N, Méreau A, Nicolas J, Tagu D and Jaubert-Possamai S, Bioinformatic prediction, deep sequencing of microRNAs and expression analysis during phenotypic plasticity in the pea aphid, Acyrthosiphon pisum. BMC Genomics 11: 281 (2010).

13. Lema $\mathrm{C}$ and Cunningham MJ, MicroRNAs and their implications in toxicological research. Toxicology letters198: 100-105 (2010).

14. Dluzen DF, Sun D, Salzberg AC, Jones N, Bushey RT, Robertson GP and Lazarus P, Regulation of UDPglucuronosyltransferase 1A1 expression and activity by microRNA 491-3p. Journal of Pharmacology and Experimental Therapeutics348: 465-477 (2014).

15. Choi YM, An S, Lee EM, Kim K, Choi SJ, Kim JS, Jang HH, An IS, Bae S, CYP1A1 is a target of miR892a-mediated post-transcriptional repression. International Journal of Oncology 41:331-336 (2012).

16. Zhu B, Xu M, Shi H, Gao X, Liang P, Genome-wide identification of IncRNAs associated with chlorantraniliprole resistance in diamondback moth Plutella xylostella (L.). BMC Genomics18: 380 (2017).

17. Papageorgiou I and Court MH, Identification and validation of the microRNA response elements in the 3'-untranslated region of the UDP glucuronosyltransferase (UGT) 2B7 and 2B15 genes by a functional genomics approach. Biochemical Pharmacology 146: 199-213 (2017).

18. Hong S, Guo Q, Wang W, Hu S, Fang F, Lv Y, Yu J, Zou F, Lei Z, Ma K, Ma L, Zhou D, Sun Y, Zhang D, Shen B, Zhu C, Identification of differentially expressed microRNAs in Culex pipiens and their potential roles in pyrethroid resistance. Insect Biochemistry and Molecular Biology 55:39-50 (2014).

19. Lei Z, Lv Y, Wang W, Guo Q, Zou F, Hu S, Fang F, Tian M, Liu B, Liu X, Ma K Ma L, Zhou D, Zhang D, Sun Y, Shen B, Zhu C, MiR-278-3p regulates pyrethroid resistance in Culex pipiens pallens. Parasitology Research 114: 699-706 (2015).

20. Chen M, Han Z, Qiao X and Qu M, Resistance mechanisms and associated mutations in acetylcholinesterase genes in Sitobion avenae (Fabricius). Pesticide Biochemistry and Physiology 87:189-195 (2007).

21. Wang $\mathrm{K}$, Zhang M, Huang Y, Yang Z, Su S and Chen M, Characterisation of imidacloprid resistance in the bird cherry-oat aphid, Rhopalosiphum padi, a serious pest on wheat crops. Pest Management Science 74:1457-1465 (2018).

22. Zhang S, Shao ZY and Li YP, Monitoring of agricultural pest resistance and suggestions for scientific medicine in 2013. China Plant Protection 34:22-25 (2014).

23. Lu Y and Gao X, Multiple mechanisms responsible for differential susceptibilities of Sitobion avenae (Fabricius) and Rhopalosiphum padi (Linnaeus) to pirimicarb. Bulletin of Entomological Research 99: 611-617 (2009). 
24. Chen X, Xia X, Wang H, Qiao K and Wang K, Cross-resistance to clothianidin and acetamiprid in the imidacloprid resistant strain of Aphis gossypii (Hemiptera: Aphididae) and the related enzyme mechanisms. Acta Entomologica Sinica 56: 1143-1151 (2013).

25. Xu LN, Ling YH, Wang YQ, Wang ZY, Hu BJ, Zhou ZY, Hu F, He KL, Identification of differentially expressed microRNAs between Bacillus thuringiensis Cry1Ab-resistant and-susceptible strains of Ostrinia furnacalis. Scientific reports5: 15461(2015).

26. Friedlander MR, Mackowiak SD, Li N, Chen W and Rajewsky N, MiRDeep2 accurately identifes known and hundreds of novel microRNA genes in seven animal clades. Nucleic Acids Research 40:37-52 (2012).

27. Enright AJ, John B, Gaul U, Tuschl T, Sander C and Marks DS, MicroRNA targets in Drosophila. Genome Biology5: R1 (2003).

28. Betel D, Wilson M, Gabow A, Marks DS, Sander C, The microRNA. org resource: targets and expression. Nucleic acids Research36: D149-D153 (2008).

29. Rehmsmeier M, Steffen $P$, Höchsmann $M$, Giegerich R, Fast and effective prediction of microRNA/target duplexes. RNA10: 1507-1517 (2004).

30. Conesa A, Götz S, García-Gómez JM, Terol J, Talón M, Robles M, Blast2GO: a universal tool for annotation, visualization and analysis in functional genomics research. Bioinformatics 21: 36743676 (2005).

31. Robinson MD, McCarthy DJ, Smyth GK, edgeR: a Bioconductor package for differential expression analysis of digital gene expression data. Bioinformatics26: 139-140 (2010).

32. Benjamini Y, Hochberg Y, Controlling the false discovery rate: a practical and powerful approach to multiple testing. Journal of the Royal statistical society: series B (Methodological)57: 289-300 (1995).

33. Ma KS, Li F, Liu Y, Liu, Y, Liang PZ, Chen XW, Gao X, Identification of microRNAs and their response to the stress of plant allelochemicals in Aphis gossypii (Hemiptera: Aphididae). BMC Molecular Biology, 18: 5 (2017).

34. Pfaffl MW, A new mathematical model for relative quantification in real-time RT-PCR. Nucleic Acids Research29: e45-e45 (2001).

35. Gong YH, Yu XR, Shang QL, Shi XY and Gao XW, 2014. Oral delivery mediated RNA interference of a carboxylesterase gene results in reduced resistance to organophosphorus insecticides in the cotton aphid, aphis gossypii Glover. PloS One 9: e102823.

36. Zhang X, Zheng Y, Jagadeeswaran G, Ren R, Sunkar R and Jiang H, Identification and developmental profiling of conserved and novel microRNAs in Manduca sexta. Insect Biochemistry and Molecular Biology42: 381-395 (2012).

37. Cai ZG, Zhang SM, Zhang H, Zhou YY, Wu HB and Xu XP, Aberrant expression of microRNAs involved in epithelial-mesenchymal transition of HT-29 cell line. Cell biology internationa/ 37: 669-674 ((2013)

38. Liu Q, Tuo W, Gao H and Zhu XQ, MicroRNAs of parasites: current status and future perspectives. Parasitology Research 107: 501-507 (2010). 
39. Truini A, Coco S, Alama A, Genova C, Sini C, Dal Bello MG,Barletta G, Rijavec E, Burrafato G, Boccardo $\mathrm{F}$ and Grossi F, Role of microRNAs in Malignant mesothelioma. Cellular and Molecular Life Sciences71: 2865-2878 (2014).

40. Li X, Zhang F, Coates B, Zhang Y, Zhou X and Cheng D, Comparative profiling of microRNAs in the winged and wingless English grain aphid, Sitobion avenae (F.)(Homoptera: Aphididae). Scientific reports 6: 35668 (2016).

41. Verma P, Augustine GJ, Ammar MR, Tashiro A and Cohen SM, A neuroprotective role for microRNA miR-1000 mediated by limiting glutamate excitotoxicity. Nature Neuroscience 18: 379-385 (2015).

42. Stark A, Kheradpour P, Parts L, Brennecke J, Hodges E, Hannon GJ, Kellis M, Systematic discovery and characterization of fly microRNAs using 12 Drosophila genomes, Genome Research 17:18651879 (2007).

43. Okabe M, Imai T, Kurusu M, Hiromi $Y$ and Okano $H$, Translational repression determines a neuronal potential in Drosophila asymmetric cell division. Nature 411: 94-98 (2001).

44. Hori S, Kaneko K, Saito TH, Takeuchi H and Kubo T, Expression of two microRNAs, ame-mir-276 and1000, in the adult honeybee (Apis mellifera) brain. Apidologie42: 89-102 (2011).

45. Zhang $X$, Zheng $Y$, Cao X, Ren R, Yu X and Jiang H, Identification and profiling of Manduca sexta microRNAs and their possible roles in regulating specific transcripts in fat body, hemocytes, and midgut. Insect Biochemistry and Molecular Biology 62: 11-22 (2015).

46. Ronshaugen M, Biemar F, Piel J, Levine M and Lai EC, The Drosophila microRNA iab-4 causes a dominant homeotic transformation of halteres to wings. Genes \& Development19: 2947-2952 (2005).

47. Garaulet DL, Castellanos MC, Bejarano F, Sanfilippo P, Tyler DM, Allan DW, Sánchez-Herrero E and Lai EC, Homeotic function of Drosophila Bithorax-complex miRNAs mediates fertility by restricting multiple Hox genes and TALE cofactors in the CNS. Developmental cel/29: 635-648 (2014).

48. Omiecinski CJ, Vanden Heuvel JP, Perdew GH and Peters JM, Xenobiotic metabolism, disposition, and regulation by receptors: from biochemical phenomenon to predictors of major toxicities. Toxicological Sciences 120: 49-75 (2011).

49. Li X, Schuler MA and Berenbaum MR, Molecular mechanisms of metabolic resistance to synthetic and natural xenobiotics. Annual Review of Entomology 52: 231-53 (2007).

50. Kaplanoglu E, Chapman P, Scott IM and Donly C, Overexpression of a cytochrome P450 and a UDPglycosyltransferase is associated with imidacloprid resistance in the Colorado potato beetle, Leptinotarsa decemlineata. Scientific Reports7: 1762. (2017)..

51. Tian F, Wang Z, Li C, Liu J and Zeng, X, UDP-Glycosyltransferases are involved in imidacloprid resistance in the Asian citrus psyllid, Diaphorina citri (Hemiptera: Lividae). Pesticide Biochemistry and Physiology 154: 23-31 (2019).

\section{Tables}

Table 1. Primers used in the study

Page $14 / 24$ 


\begin{tabular}{|c|c|c|c|}
\hline Primer name & Sequence $\left(5^{\prime}-3^{\prime}\right)$ & Size $(b p)$ & Application \\
\hline \multirow{2}{*}{ api-mir-1000 } & F:AGAGCACGATATTGTCCTGTCACA & \multirow[t]{2}{*}{73} & qPCR \\
\hline & R:TATGGTTGTTCACGACTGGTTCAC & & qPCR \\
\hline \multirow[t]{2}{*}{ api-mir-316 } & F:TTTGGTGTCTTTTTCCGCTTTG & \multirow[t]{2}{*}{69} & qPCR \\
\hline & R:GTGCAGGGTCCGAGGT & & qPCR \\
\hline \multirow[t]{2}{*}{ api-mir-iab-4 } & F:AGGCGAAGCGACGTATACTAAATG & \multirow[t]{2}{*}{68} & qPCR \\
\hline & R:GTGCAGGGTCCGAGGT & & qPCR \\
\hline \multirow[t]{2}{*}{ U6 } & F:CAGCACATATACTAAAATTGGAACG & \multirow[t]{2}{*}{76} & qPCR \\
\hline & R:ACGAATTTGCGTGTCATCC & & qPCR \\
\hline MicroRNA inhibitor N.C & CAGUACUUUUGUGUAGUACAA & 21 & miRNA function \\
\hline api-mir-1000 inhibitor & UACUGCUGUGACAGGACAAUAU & 22 & miRNA function \\
\hline api-mir-316 inhibitor & CGGCAGCAAAGCGGAAAAAGACA & 23 & miRNA function \\
\hline api-mir-iab-4 inhibitor & UCAGGAUACAUUUAGUAUACGU & 22 & miRNA function \\
\hline \multirow[t]{2}{*}{ CYP6A14 } & F: TGTGCACCAAACAGTTCAGG & 107 & qPCR \\
\hline & R:CGGATAGTACTGCGGGTCATG & & qPCR \\
\hline \multirow[t]{2}{*}{ Actin } & F:GATGCACCTGGTCACAGAGA & & qPCR \\
\hline & R:CCATCTTGTTCACACCAACG & & qPCR \\
\hline
\end{tabular}

Table 2. Distribution of miRNA reads from control and imidacloprid-treated Sitobion avenae

\begin{tabular}{ccc}
\hline Parameter & Control & Imidacloprid treatment \\
\hline Raw reads & $14,841,584$ & $13,964,533$ \\
Clean reads & $14,224,837$ & $13,492,898$ \\
3ADT and length filter & $10,959,929$ & $9,305,973$ \\
rRNA & 253,777 & 247,747 \\
tRNA & 16 & 23 \\
snoRNA & 9,443 & 7,823 \\
snRNA & 25,993 & 24,246 \\
Mappable reads & $6,992,451$ & $6,349,363$ \\
\hline
\end{tabular}

Note: 3ADT: reads that were removed because 3ADT was not found and length was less than $15 \mathrm{nt}$ or greater than $35 \mathrm{nt}$. Mappable reads: the number of raw reads that passed through the many digital filters .

Table 3. The differentially expressed miRNAs in the small RNA libraries of Sitobion avenae 


\begin{tabular}{|c|c|c|c|c|c|}
\hline miR_Name & $\begin{array}{l}\text { Imidacloprid } \\
\text { treatment_FPKM }\end{array}$ & Control_FPKM & $\begin{array}{l}\mathrm{P}- \\
\text { value }\end{array}$ & $\begin{array}{l}\log _{2}(\text { Fold } \\
\text { Change) }\end{array}$ & Difference \\
\hline api-miR-998 & 4021.337154 & 14561.84926 & 0 & -1.8564 & Down \\
\hline Novel-28 & 435.4314115 & 2094.17899 & $\begin{array}{l}1.93 E- \\
286\end{array}$ & -2.2659 & Down \\
\hline api-miR-306 & 691.567536 & 2386.390012 & $\begin{array}{l}1.54 \mathrm{E}- \\
246\end{array}$ & -1.7869 & Down \\
\hline Novel-22 & 742.7947608 & 1801.967968 & $\begin{array}{l}1.83 \mathrm{E}- \\
120\end{array}$ & -1.2785 & Down \\
\hline Novel-9 & 717.1811484 & 1704.564294 & $\begin{array}{l}1.79 \mathrm{E}- \\
110\end{array}$ & -1.249 & Down \\
\hline api-miR-3047 & 332.9769618 & 779.2293917 & $\begin{array}{l}3.01 \mathrm{E}- \\
50\end{array}$ & -1.2266 & Down \\
\hline Novel-25 & 256.1361244 & 657.4747992 & $\begin{array}{l}6.09 \mathrm{E}- \\
49\end{array}$ & -1.36 & Down \\
\hline api-miR-1000 & 128.0680622 & 0 & $\begin{array}{l}8.16 \mathrm{E}- \\
28\end{array}$ & 8.0008 & Up \\
\hline api-miR-316 & 0 & 48.70183698 & $\begin{array}{l}2.37 \mathrm{E}- \\
13\end{array}$ & -6.6059 & Down \\
\hline Novel-39 & 51.22722489 & 0 & $\begin{array}{l}4.65 E- \\
13\end{array}$ & 6.6788 & Up \\
\hline Nove-35 & 153.6816747 & 48.70183698 & $\begin{array}{l}2.31 \mathrm{E}- \\
12\end{array}$ & 1.6579 & Up \\
\hline api-miR-263b & 0 & 24.35091849 & $\begin{array}{l}1.57 \mathrm{E}- \\
07\end{array}$ & -5.6059 & Down \\
\hline api-miR-2796 & 0 & 24.35091849 & $\begin{array}{l}1.57 \mathrm{E}- \\
07\end{array}$ & -5.6059 & Down \\
\hline api-miR-iab-4 & 0 & 24.35091849 & $\begin{array}{l}1.57 \mathrm{E}- \\
07\end{array}$ & -5.6059 & Down \\
\hline $\begin{array}{l}\text { api-miR- } \\
3015 a\end{array}$ & 25.61361244 & 0 & $\begin{array}{l}2.52 \mathrm{E}- \\
07\end{array}$ & 5.6788 & Up \\
\hline api-miR-3042 & 76.84083733 & $\begin{array}{r}24.35091849 \\
\text { Page 16/24 }\end{array}$ & 7.05E- & 1.6579 & Up \\
\hline
\end{tabular}


Table 4 MiRNAs targeting putative genes related to xenobiotic metabolism in Sitobion avenae 


\begin{tabular}{|c|c|}
\hline miR_Name & Target genes related to xenobiotic metabolism \\
\hline api-miR- & S-(hydroxymethyl)glutathione dehydrogenase / alcohol dehydrogenase \\
\hline 998 & CYP3A19, glutathione S-transferase omega-1, glutathione S-transferase, C-terminal domain \\
\hline novel-28 & $\begin{array}{l}\text { Microsomal epoxide hydrolase, glutathione S-transferase, C-terminal domain, P450 (GC vitamin D- } \\
\text { binding protein), CYP4C1, CYP4V, CYP9Z4 }\end{array}$ \\
\hline api-miR- & alcohol dehydrogenase 4, UDP-glucuronosyltransferase 2B10, UDP-glucuronosyltransferase 2B7, \\
\hline 306 & $\begin{array}{l}\text { Cytochrome P450 (piezo-type mechanosensitive ion channel component 2), CYP4C1, Glutathione S- } \\
\text { transferase (EEF1G elongation factor 1-gamma), CYP6A14 }\end{array}$ \\
\hline novel-22 & $\begin{array}{l}\text { UDP-glucuronosyltransferase 2C1, UDP-glucuronosyltransferase 1-7C UDP-glucuronosyltransferase } \\
\text { 2B17, Glutathione S-transferase Mu 1, Glutathione S-transferase (phosphatidylinositol-binding clathrin } \\
\text { assembly protein LAP), CYP4g15, CYP4C1 }\end{array}$ \\
\hline novel-9 & $\begin{array}{l}\text { UDP-glucuronosyltransferase 2C1, carbonyl reductase 1, Microsomal glutathione S-transferase 1, UDP- } \\
\text { glucuronosyltransferase 1-7 }\end{array}$ \\
\hline $\begin{array}{l}\text { api-miR- } \\
3047\end{array}$ & $\begin{array}{l}\text { UDP-glucuronosyltransferase 2C1, UDP-glucuronosyltransferase 1-7, CYP315A1, CYP2U1, P450 (piezo- } \\
\text { type mechanosensitive ion channel component 2) }\end{array}$ \\
\hline novel-25 & $\begin{array}{l}\text { alcohol dehydrogenase class-3, S-(hydroxymethyl)glutathione dehydrogenase / alcohol dehydrogenase, } \\
\text { glutathione S-transferase, C-terminal domain, CYP3A19, }\end{array}$ \\
\hline api-miR- & UDP-glucuronosyltransferase 2B7, UDP-glucuronosyltransferase 2B10, UDP-glucuronosyl transferase \\
\hline 1000 & $\begin{array}{l}\text { 351A3, Glutathione S-transferase (phosphatidylinositol-binding clathrin assembly protein LAP), } \\
\text { Glutathione S-transferase (failed axon connections), Cytochrome P450 (piezo-type mechanosensitive ion } \\
\text { channel component 2), CYP4C1, CYP6A2, CYP6A13 }\end{array}$ \\
\hline $\begin{array}{l}\text { api-miR- } \\
316\end{array}$ & $\begin{array}{l}\text { UDP-glucuronosyltransferase 2B1, Cytochrome P450 (piezo-type mechanosensitive ion channel } \\
\text { component 2), CYP4C1, CYP305a1, Cytochrome bd terminal oxidase }\end{array}$ \\
\hline novel-39 & Glutathione S-transferase GSTZ1 \\
\hline novel-35 & $\begin{array}{l}\text { Glutathione S-transferase (phosphatidylinositol-binding clathrin assembly protein LAP), Cytochrome } \\
\text { P450 (piezo-type mechanosensitive ion channel component 2), CYP49A1, CYP6A14, CYP315A1, } \\
\text { CYP4C1, CYP6A13, CYP6J1 }\end{array}$ \\
\hline $\begin{array}{l}\text { api-miR- } \\
263 \mathrm{~b} \\
\text { api-miR- } \\
2796\end{array}$ & $\begin{array}{l}\text { UDP-glucuronosyltransferase 2C1, UDP-glucuronosyltransferase 2A1, glutathione S-transferase C- } \\
\text { terminal domain, CYP6A13, CYP4C1, CYP4V2, CYP49A1, } \\
\text { UDP-glucuronosyltransferase 2B18 }\end{array}$ \\
\hline $\begin{array}{l}\text { api-miR- } \\
\text { iab-4 }\end{array}$ & CYP314A1, CYP4C1, CYP6A13, CYP3A19 \\
\hline api-miR- & alcohol dehydrogenase class-3, UDP-glucuronosyltransferase 2A1 dehydrogenase, glutathione S- \\
\hline $3015 a$ & $\begin{array}{l}\text { transferase C-terminal domain, Glutathione S-transferase (phosphatidylinositol-binding clathrin } \\
\text { assembly protein LAP), Cytochrome P450 (piezo-type mechanosensitive ion channel component 2), } \\
\text { CYP3A19, }\end{array}$ \\
\hline api-miR- & Glutathione S-transferase (EEF1G elongation factor 1-gamma) \\
\hline 3042 & \\
\hline
\end{tabular}




\section{Additional Information}

Table S1. Toxicity of imidacloprid on Sitobion avenae

Table S2. Summary of conserved miRNAs.

Table S3.Summary of predicted miRNAs.

Table S4. The predicted targets of miRNAs in Sitobion avenae.

Table S5. GO annotations for identified Sitobion avenae miRNA targets.

Table S6. KEGG analysis of targets of differentially expressed Sitobion avenae miRNAs.

Fig. S1. Predicted stem-loop structures for three novel miRNA precursors.

Fig. S2. qPCR validation of miRNAs potentially involved in resistance of Sitobion avenae to imidacloprid.

\section{Figures}
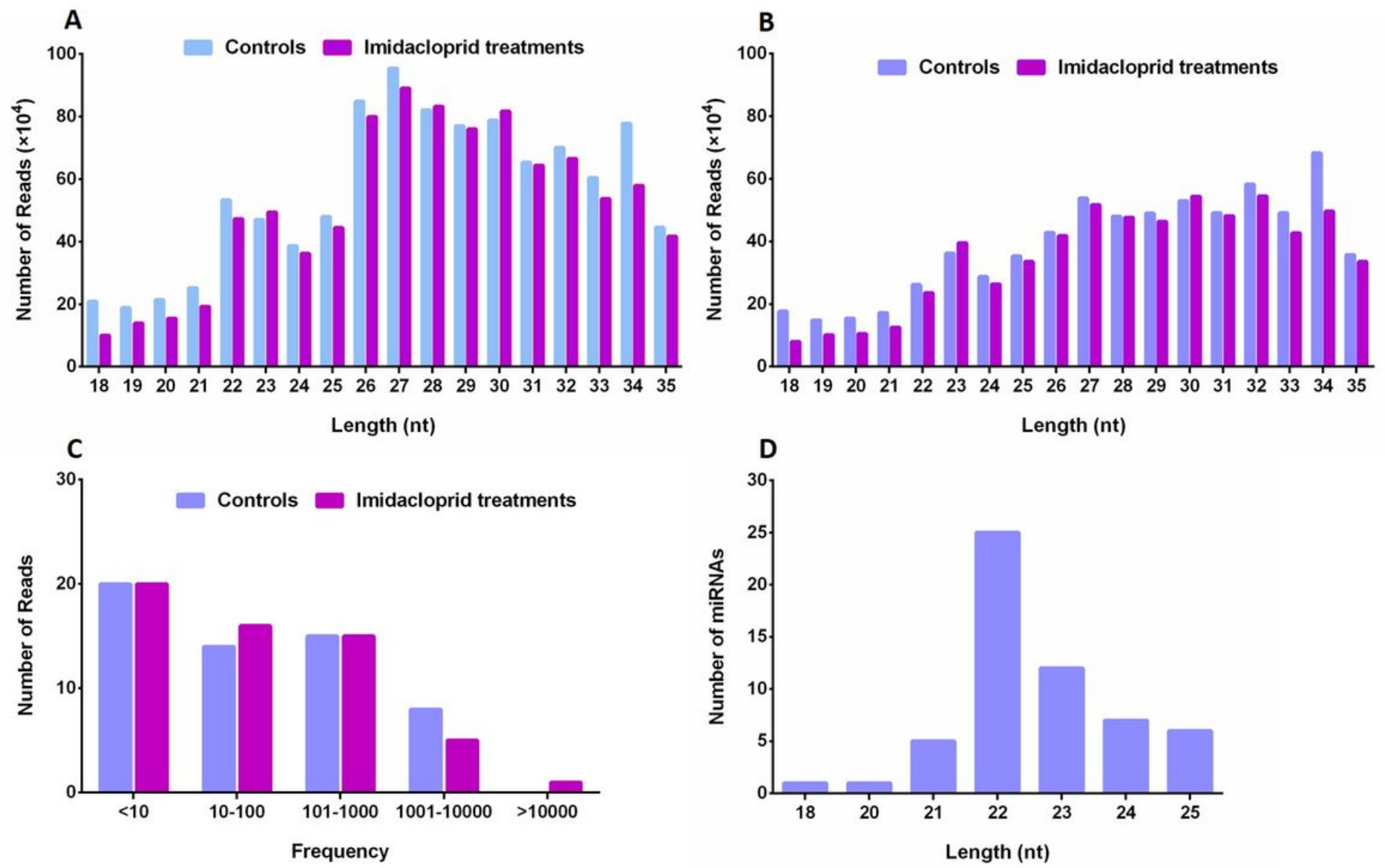

Figure 1 
Characterization of miRNA sequences from S. avenae. A: Length distribution of raw reads, B: length distribution of mappable reads, C: frequency distribution of read counts, and D: length distribution of miRNAs.

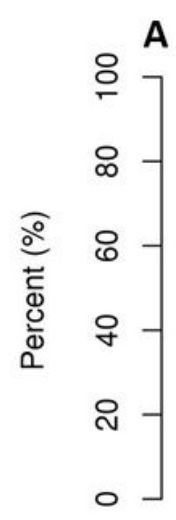

miRNA Nucleotide Bias at Each Position (APN2)
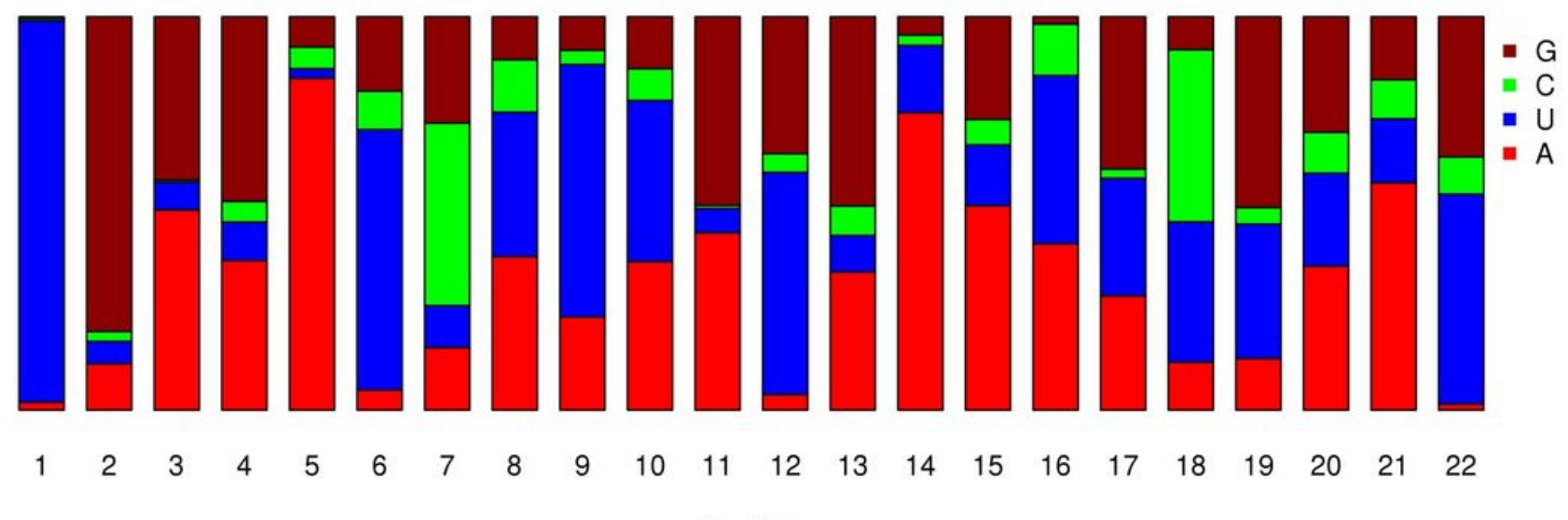

Position

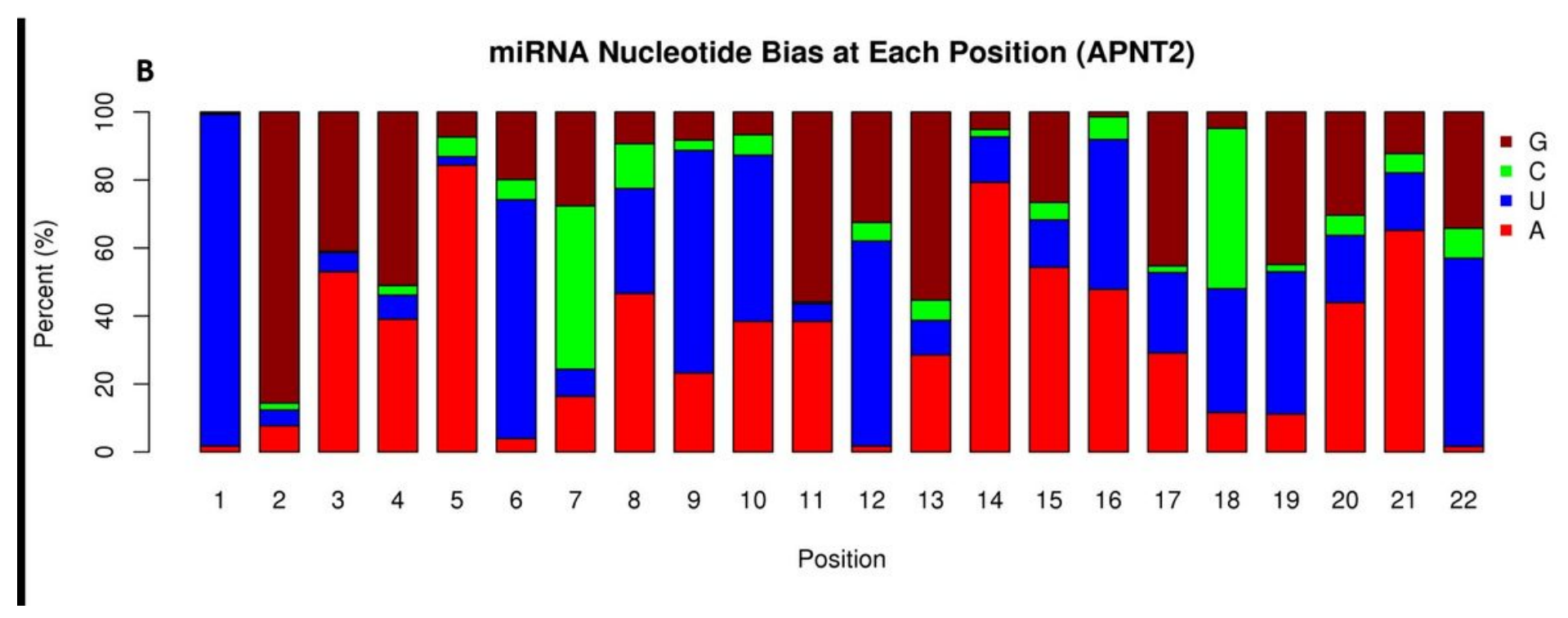

Figure 2

Nucleotide bias of predicated miRNAs from Sitobion avenae. 

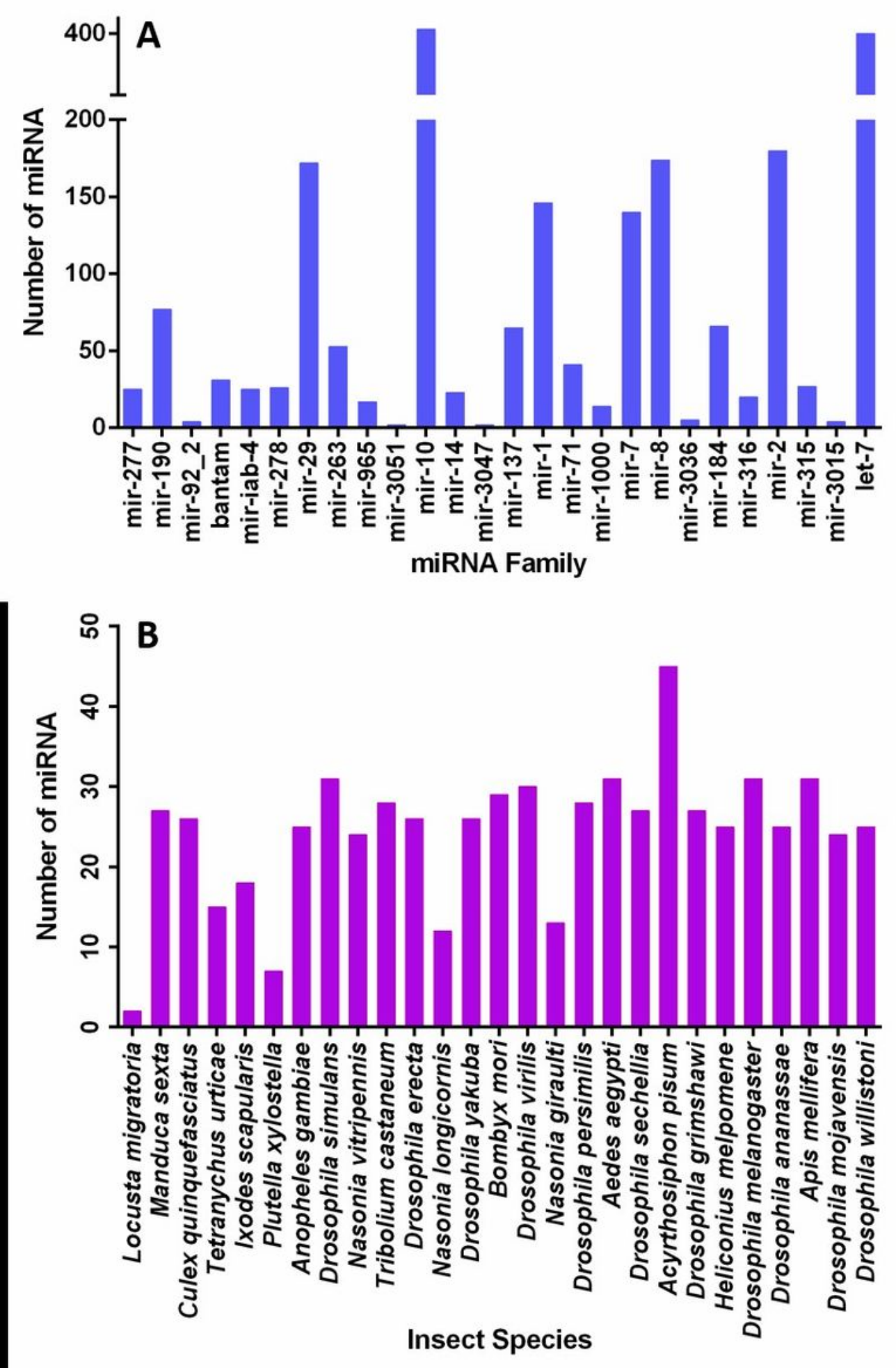

Figure 3

MiRNAs characterization of Sitobion avenae. 


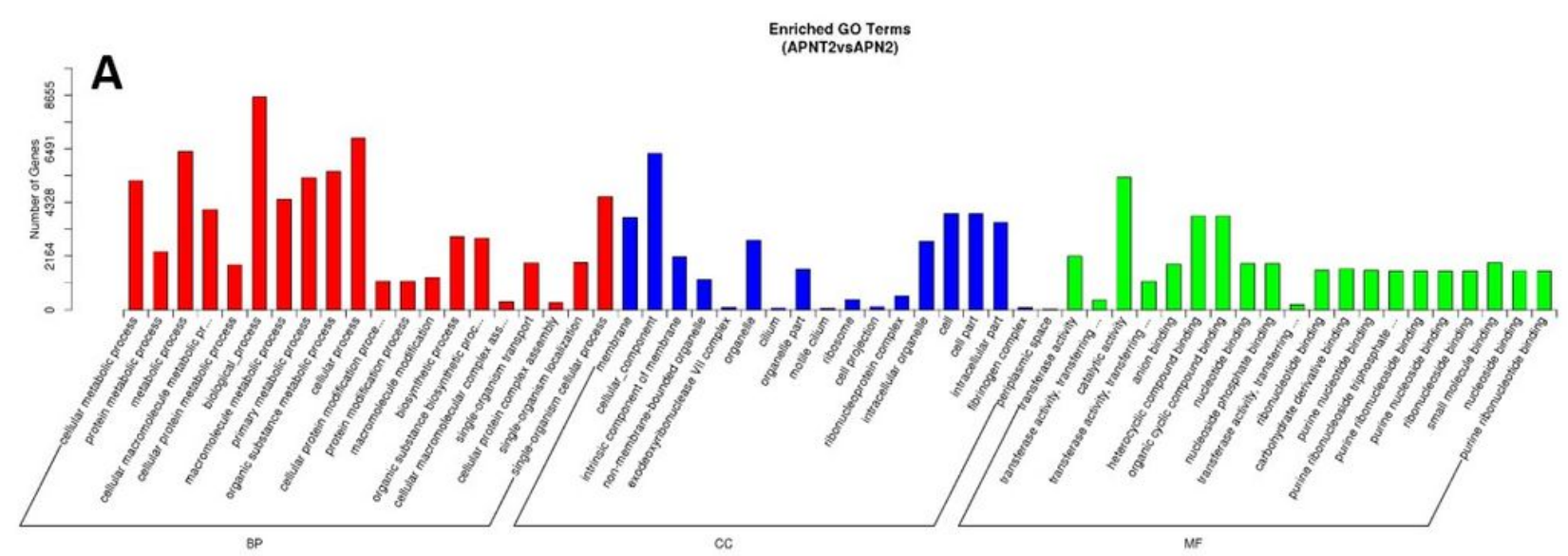

B

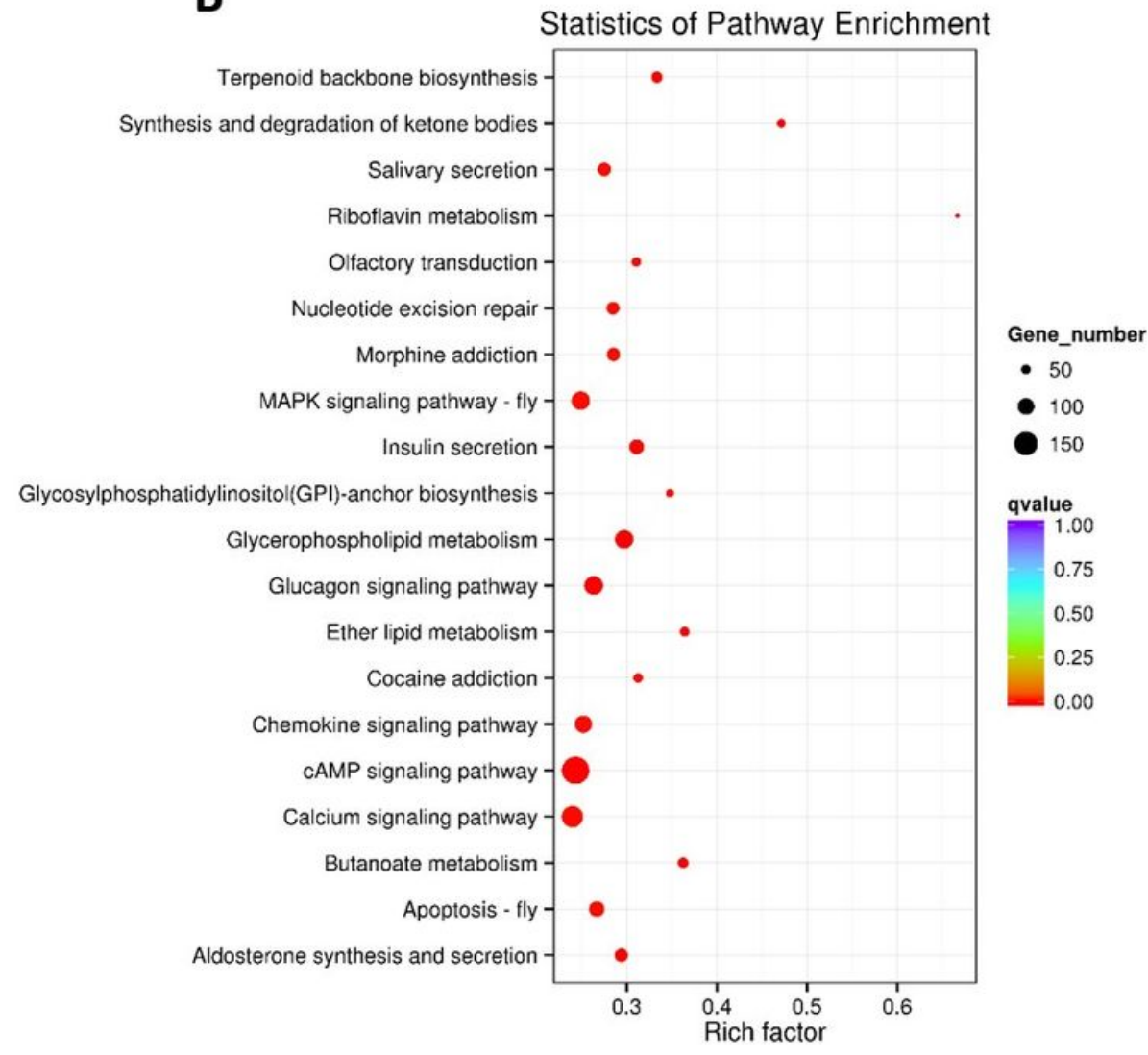

Figure 4

Histogram presentation of GO annoation and KEGG pathway for miRNAs identified from Sitobion avenae. 

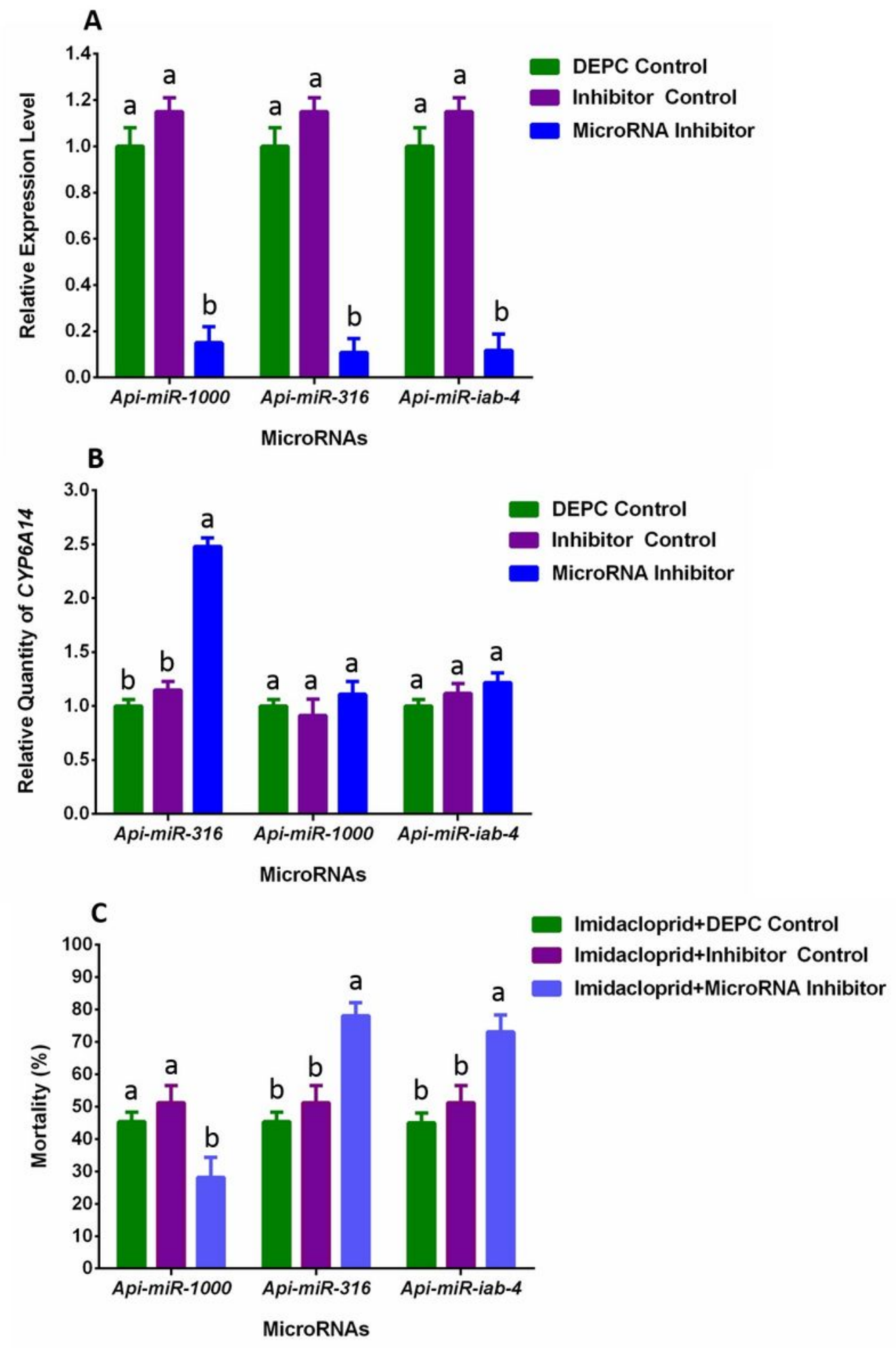

\section{Figure 5}

Effects of miRNA modulation on CYP6A14 expression and imidacloprid susceptibility. (A) Orally mediated inhibition of miRNAs; (B) Effect of inhibition of the api-miR-1000, api-miR-316, and api-miR-iab4 miRNAs on the expression of CYP6A14; (C) The effect of modulating the levels of api-miR-1000, apimiR-316, and api-miR-iab-4 using feeding inhibitors on imidacloprid tolerance in S. avenae. Mean mortality \pm SE. Error bars indicate $95 \%$ confidence intervals. Different letters on the bars of the histogram 
indicate significant differences based on ANOVA followed by Tukey's HSD multiple comparison test $(\mathrm{P}<$ 0.05).

\section{Supplementary Files}

This is a list of supplementary files associated with this preprint. Click to download.

- TablesSupp.xlsx

- File1.zip

- FigsSupp.pdf 